\title{
The effect of metallicity on the Cepheid period-luminosity relation
}

\author{
Martino Romaniello, Francesca Primas, Marta Mottini \\ European Southern Observatory, Karl-Schwarzschild-Straße 2, D-85748 \\ Garching bei München, Germany \\ Martin Groenewegen \\ Instituut voor Sterrenkunde, Celestijnenlaan 200B, B-3001 Leuven, \\ Belgium
}

\begin{abstract}
We present preliminary results of an observational campaign devoted to establishing the influence of chemical composition on the Cepheid Period-Luminosity relation. The data are in good agreement with theoretical predictions based on non-linear convective models, suggesting a fairly strong dependence of the Period-Luminosity relation on metallicity in the sense of more metal rich stars being intrinsically fainter than otherwise expected. Our data indicate that the error on the inferred distance can be as large as $10 \%$ if the role of metallicity is neglected.
\end{abstract}

\section{Introduction}

Ever since the work of Edwin Hubble, the Cepheid Period-Luminosity (PL) relation has been a fundamental tool in determining Galactic and extragalactic distances. In spite of its paramount importance, to this day we still lack firm theoretical and empirical assessment on whether or not chemical composition has any significant influence on the pulsational properties of Cepheids.

The debate on metallicity effects on the PL relation is an old one (see, for example, the reviews by Madore \& Freedman 1991 and, more recently, Feast 2003), but the matter is far from being settled. As an illustration, different prescriptions on how to account for the metallicity effect lead to differences as large as $10 \%$ in the distance to the Large Magellanic Cloud (e.g., Groenewegen \& Oudmaijer 2000), which directly translates into a $10 \%$ uncertainty on $H_{0}$. To make things worse, this effect is systematic and only a careful calibration of the dependence of the PL relation on metallicity can provide firmer ground on which to build the astronomical distance ladder. Regrettably, the current understanding of the subject is poor.

Theoretical pulsational models by different groups lead to markedly different results. On the one side computations based on linear models (e.g., Chiosi et al. 1992; Sandage et al. 1999; Alibert et al. 1999; Baraffe \& Alibert 2001) suggest a mild dependence of the PL relation on chemical composition (less than $0.1 \mathrm{mag}$ at $\log (P)=1$ for a change in metallicity between $Z=0.004$ and $Z=0.02$ ). This result is challenged by the outcome of the non-linear convective models 
(e.g. Bono et al. 1999; Caputo et al. 2000) which find that both the slope and the zeropoint of the PL relation depend significantly on the adopted chemical composition. Again for $\log (P)=1$ and the same variation in metallicity as above, they predict a change as large as $0.4 \mathrm{mag}$ in $V, 0.3 \mathrm{mag}$ in $I$ and $0.2 \mathrm{mag}$ in $K$. Moreover, the change is such that metal-rich Cepheids are fainter than metal-poor ones, again at variance with the results of Alibert et al. (1999) and Baraffe \& Alibert (2001). Finally, recent calculations by Fiorentino et al. (2002) based on non-linear models indicate that the PL relation also depends on the helium content of the stars.

From an observational standpoint, the subject was approached in essentially two ways: a direct measurement of the iron content in nearby, i.e. bright, Cepheids, or by measuring a secondary metallicity indicator in external galaxies known to contain Cepheids, under the assumption that they would have the same chemical composition. Regrettably, direct metallicity determinations for individual Cepheids exist only for a handful of stars. The most accurate studies are by Fry \& Carney $(1997,[\mathrm{Fe} / \mathrm{H}]$ and $[\alpha / \mathrm{H}]$ for 23 Galactic Cepheids) and Andrievsky et al. (2002, complete abundance analysis of 77 Galactic Cepheids; see also Luck et al. 2003). The former find a spread in $[\mathrm{Fe} / \mathrm{H}]$ of $0.4 \mathrm{dex}$, which they argue is real, the latter focus on investigating Galactic abundance gradients $\left(-0.05 \mathrm{dex} \mathrm{kpc}^{-1}\right)$. Luck et al. (1998) have measured the iron abundance of 16 Cepheids in the Large and Small Magellanic Cloud (LMC and SMC, respectively) with the echelle spectrograph at the CTIO 4-m telescope. The relatively small size of the telescope forced them to choose only stars at the bright end of the $\mathrm{PL}$ relation, i.e. long periods, whereas the entire range needs to be sample in order to reach meaningful conclusions on the PL relation (e.g., Kennicut et al. 1998; Caputo et al. 2000). As for the indirect measurements in external galaxies (e.g., Kochanek et al. 1997; Kennicutt et al. 1998), which probe a broader range in metallicity than the one available in the Galaxy, they find that metal-rich Cepheids are brighter in $V$ and $I$ than metal-poor ones by 0.24 to $0.40 \mathrm{mag}$ per dex in metallicity. These results have been widely used in the literature, including in the final paper of the HST Key Project on $H_{0}$ (Freedman et al. 2001).

\section{The data sample}

With the scenario described above in mind, we have collected high quality spectra of an unprecedented data set of 40 Galactic, 22 LMC and 14 SMC Cepheids with the aim of measuring metallicity directly on a statistically significant sample of stars covering about one order of magnitude in chemical composition. The Galactic stars were observed with FEROS at the ESO 1.5- $\mathrm{m}$ telescope on La Silla at a spectral resolution of 48000 with a signal-to-noise ratio of about 70 to 150. As for the spectra of the Magellanic Cloud objects, we used the UVES spectrograph on the Kueyen telescope on Cerro Paranal, yielding a resolution of 40000 and signal-to-noise ratio of about 50 to 70 .

Deriving metallicities for Cepheids is by no means a trivial task, but we have now carefully analyzed and derived $[\mathrm{Fe} / \mathrm{H}]$ for an initial subsample of 14 Cepheids in the LMC, 12 in the SMC and 6 in the Galaxy. The data reduction procedures and analysis are described in detail in Mottini et al. (2003). 


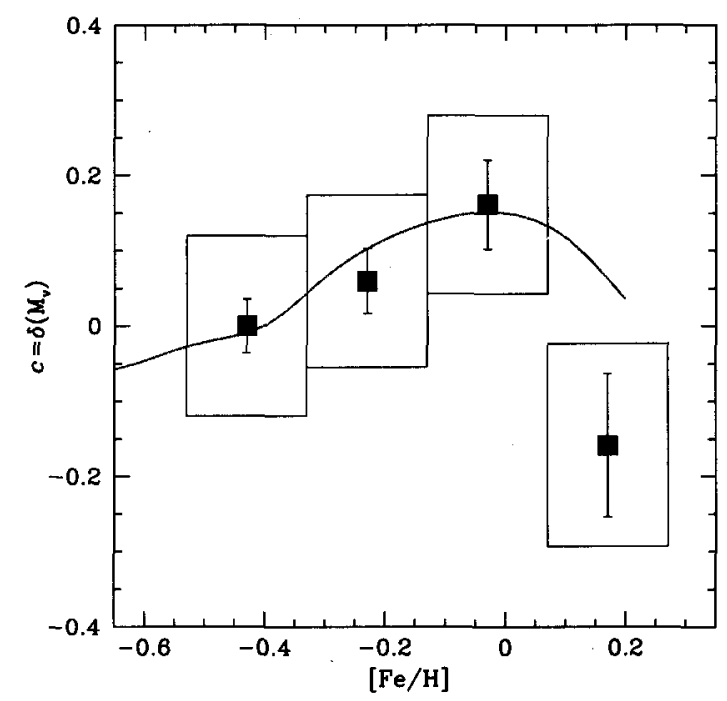

Figure 1. $\quad V$-band residuals compared to the Madore \& Freedman (1991) PL relation vs the iron content we have measured from our UVES and FEROS spectra (14 stars in the LMC, 12 in the SMC and 6 in the Galaxy). Filled squares represent the median value in each metallicity bin with its associated errorbar and the rms in each bin is displayed as boxes. The solid line shows the predictions by Fiorentino et al. (2002).

\section{Results: dependence of the PL relation on metallicity}

The main result from the subset analyzed so far is summarized in Fig. 1. There we plot the $V$-band residuals from the standard Madore \& Freedman (1991) PL relation as a function of the iron abundance derived from FEROS and UVES spectra. A positive magnitude difference here means that the star is fainter than the mean PL relation. For consistency with the adopted PL relation, the LMC is assumed to have a distance modulus of 18.50. The SMC is considered $0.44 \mathrm{mag}$ more distant (e.g. Cioni et al. 2000) and the distances to Galactic stars were taken from Fouqué et al. (2003), Barnes et al. (2003) and Tammann et al. (2003). Photometry and reddening are taken from Laney \& Stobie (1994).

The data are binned in metallicity to reflect the typical observational error on our determination $( \pm 0.1 \mathrm{dex})$. The Magellanic Cloud Cepheids populate the bins at lower metallicity, the higher $[\mathrm{Fe} / \mathrm{H}]$ one containing only Galactic stars. The median magnitude residual in each bin is plotted as a square and the rms and error on the median are shown as boxes and errorbars, respectively. The magnitude difference plotted on the ordinates can be directly compared to the "c" quantity computed by Fiorentino et al. (2002). This is the correction to be applied to the distance modulus derived using a universal, metallicity independent PL relation to take into account the influence of metallicity as derived from their non-linear pulsation models. 
The agreement between our data and the predictions by Fiorentino et al. (2002), indicating a strong dependence of the PL relation on metallicity, is tantalizing, even though the errorbars are still somewhat large. As it can be seen, the error on the inferred distance can be as large as $10 \%$ if the effect of metallicity is neglected.

\section{References}

Alibert, Y., et al. 1999, A\&A344, 551

Andrievsky, S.M., et al. 2002, A\&A, 381, 32

Baraffe, I., Alibert, Y. 2001, A\&A, 371, 592

Barnes, T.G., et al. 2003, ApJ, 592, 539

Bono, G., et al. 1999, ApJ, 512, 711

Caputo, F., et al. 2000, A\&A, 359, 1059

Chiosi, C., et al. 1992, ApJ, 387, 320

Cioni, M.-R., et al. 2000, A\&A, 359, 614

Feast, M. 2003, in Stellar Candles for the Extragalactic Distance Scale, Lect. Notes Phys., 635, 45

Fiorentino, G., et al. 2002, ApJ, 576, 402

Fouqué, P., et al. 2003 in Stellar Candles for the Extragalactic Distance Scale, Lect. Notes Phys., 635, 21

Fry, A.M., Carney, B.W. 1997, AJ, 113, 1973

Groenewegen, M., Oudmaijer, R.D. 2000, A\&A, 356, 849

Kennicutt, R.C., et al. 1998, ApJ, 498, 181

Kochanek, C.S. 1997, ApJ, 491, 13

Laney, C.D., Stobie, R.S. 1994, MNRAS, 266, 441

Luck, R.E., et al. 1998, AJ, 115, 605

Luck, R.E., et al. 2003, A\&A, 401, 939

Madore, B., Freedman, W. 1991, PASP, 103, 933

Mottini, M., et al. 2003, this conference

Sandage, A., et al. 1999, ApJ, 522, 250

Tammann, G., et al. 2003, A\&A, 404, 423 